\title{
Does Low Surface Brightness Mean Low Density?
}

\author{
W.J.G. de Blok \\ Kapteyn Astronomical Institute \\ P.O. Box 800 \\ 9700 AV Groningen \\ The Netherlands \\ and \\ S.S. McGaugh \\ Department of Terrestrial Magnetism \\ Carnegie Institution of Washington \\ 5241 Broad Branch Road NW \\ Washington, DC 20015
}

\begin{abstract}
We compare the dynamical properties of two galaxies at identical positions on the Tully-Fisher relation, but with different surface brightnesses. We find that the low surface brightness galaxy UGC 128 has a higher mass-to-light ratio, and yet has lower mass densities than the high surface brightness galaxy NGC 2403. This is true for the gas surface density, the stellar surface density, and the total mass density.

Subject headings: galaxies: individual (NGC 2403, UGC 128) - galaxies: fundamental parameters - galaxies: structure - galaxies: spiral - galaxies: kinematics and dynamics - dark matter
\end{abstract}




\section{Introduction}

Rotation curves of disk galaxies are observed to remain flat at large radii, and do not show the Keplerian decline expected on basis of the visible matter. This is usually explained by dark matter (DM) that surrounds the visible parts of these galaxies. This DM is generally assumed to be in the form of a massive halo, which stabilizes the embedded visible disk and dominates the gravitational field in the outer parts.

Motivated by the observations that the properties of the visible components of galaxies change along the Hubble sequence (see Roberts \& Haynes 1994), attempts have been made to find out whether the properties of the DM halos also change systematically with galaxy type or other parameters (e.g. Tinsley 1981, Athanassoula, Bosma \& Papaioannou 1987, Kormendy 1990, Broeils 1992). A general conclusion is that the importance of DM increases from early towards late-type galaxies.

It is interesting to know how the DM fraction depends on physical properties like galaxy luminosity, disk size and surface brightness. In general, it is believed that luminosity correlates with the total mass of the halo as it should if the baryonic mass fraction and mass to light ratio are not strongly variable. Here, we wish to address whether the mass distribution also follows that of the light. This contains two separate questions: 1) does the baryonic mass surface density follow in the obvious way from the surface brightness, and 2) is the dark matter distribution related to that of the light.

The latter question can already be answered in the affirmative from the Tully-Fisher (TF) relation (Zwaan et al. 1995). Here, we confirm this with rotation curve decompositions of two galaxies selected for their nearly identical location in the TF plane, but with very different surface brightnesses. This choice is made to isolate galaxies whose gross properties are similar, so that differences in their light distribution have testable implications for their mass distribution.

\section{The data}

Two galaxies which occupy identical positions on the TF relation are NGC 2403 and UGC 128. Both have almost identical luminosities $\left(M_{B} \simeq\right.$ $-19,\left[H_{0}=75 \mathrm{~km} \mathrm{~s}^{-1} \mathrm{Mpc}^{-1}\right.$ throughout this paper]), and almost identical maximum rotation velocities $\left(V_{\max } \simeq 134 \mathrm{~km} \mathrm{~s}^{-1}\right)$. They are morphologically very similar, but have a large difference in surface brightness. NGC 2403 has a central surface brightness of $21.4 B$-mag $\operatorname{arcsec}^{-2}$, and is therefore representative of the group of galaxies that obeys Freeman's Law (Freeman 1970). The central surface brightness of UGC 128 is $24.2 B$-mag $\operatorname{arcsec}^{-2}$. It belongs to the group of LSB disk galaxies that have been extensively described in van der Hulst et al. (1993), McGaugh \& Bothun (1994), de Blok, van der Hulst \& Bothun (1995) and de Blok, McGaugh \& van der Hulst (1996). The central surface brightness values are corrected towards face-on assuming the disks are optically thin. As both galaxies have almost identical inclinations $\left(60^{\circ}\right.$ for NGC 2403 and $57^{\circ}$ for UGC 128) any systematic error in these corrections will affect both surface brightnesses equally. The data from NGC 2403 were taken from Begeman (1987) and Kent (1987), those for UGC 128 from van der Hulst et al. (1993). NGC 2403 is at a distance of $3.3 \mathrm{Mpc}$, while UGC 128 is at $60 \mathrm{Mpc}$. Both galaxies have well-defined rotation curves; that of NGC 2403 was derived with a beam of $45^{\prime \prime} \times 45^{\prime \prime}$, while the rotation curve of UGC 128 was derived using a beam of $40^{\prime \prime} \times 40^{\prime \prime}$. We will discuss possible effects of the difference in physical resolution between the two curves later in this paper.

\section{Results}

\subsection{Disk-halo decomposition}

The structural properties of DM halos are usually derived by computing the rotation curves of the visible mass components and subtracting 
these from the observed curve. After assuming a shape for the halo (e.g. a pseudo-isothermal sphere), a fit is made to the residuals of the rotation curve. This is, however, not an unambiguous procedure. As only the distribution of the light of the stellar component is observed, and the mass-to-light ratio of the stellar component $\Upsilon_{*}$ is not known a priori, a value for $\Upsilon_{*}$ has to be assumed before the halo can be fitted. The fitted halo parameters depend strongly on this value. One way to circumvent this problem is by using the "maximum disk hypothesis" (van Albada \& Sancisi 1986). $\Upsilon_{*}$ and the rotation curve of the stellar component are scaled up to the maximum value $\Upsilon_{*}^{\max }$ allowed by the observed total rotation curve.

It is still a point of discussion whether this maximum disk solution is a physical solution (see e.g. Kuijken \& Gilmore 1989, Athanasoula et al. 1987, Bottema 1995). In many cases the surface density of the visible matter derived using other methods is found to be smaller than that implied by the maximum disk solution. The maximum disk solution is in this respect a "minimum halo" solution: surplus rotation velocity resulting from $\mathrm{DM}$ in the visible parts of galaxies is incorporated into the rotation curve of the stellar disk by increasing its $\Upsilon_{*}$.

\subsection{Results using maximum disk}

Figure 1 shows the observed rotation curves of HSB galaxy NGC 2403 and LSB galaxy UGC 128 , together with the rotation curves of the individual mass components (gas, stars, DM), derived from a maximum disk decomposition assuming an isothermal halo. The parameters of the fit are tabulated in the top panel of Table 1. Though the asymptotic velocities are the same, the rotation curve of UGC 128 rises less quickly than that of NGC 2403 (as would be expected if the mass is distributed like the light). At fixed global properties $L$ and $V_{\max }$ the shape of the rotation curves thus differs with surface brightness. Note that in UGC 128 the contributions of the gas and the stars are of equal importance in the outer parts. The gas in UGC 128 is dynamically more important than the gas in NGC 2403. In NGC 2403 the maximum stellar disk dominates over the gas everywhere, despite the fact that $\Upsilon_{*}^{\max }$ is a factor of $\sim 2$ lower than that of UGC 128. The maximum disk masses of the stellar component are approximately equal. The larger scale length of the disk of UGC 128 makes the mass distribution more diffuse, so its peak is lower and occurs at a larger radius, thus allowing large values of $\Upsilon_{*}^{\max }$ even with the more shallow total rotation curve.

Taking these large values of $\Upsilon_{*}^{\max }$ at face-value implies either a truly high stellar mass-to-light ratio or the existence of an additional dark component distributed like the stars. We can exclude the former possibility as all other evidence points towards LSB galaxies having unevolved disks. For an exponentially decreasing star formation rate and a stellar population with $B-V \sim 0.7$ (typical for a Freeman disk galaxy) population synthesis models (Larson \& Tinsley 1977) predict a stellar mass-to-light ratio $\Upsilon_{*}^{B} \simeq 2.6$, while for $B-V \sim 0.5$ (typical for LSB galaxies) $\Upsilon_{*}^{B} \simeq 0.9$. This is opposite to the trend derived from the maximum disk fits. $\Upsilon_{*}^{\max }$ in LSB galaxies is unlikely to be representative of the stellar population.

The conclusion is that DM must dominate in UGC 128 down to a small radius. It is therefore not "maximum disk" in the sense usually applied to HSB galaxies, where the inner part of the rotation curve can be completely explained by the luminous matter. This is true of LSB galaxies generally (de Blok et al. 1996): DM is needed almost everywhere to explain the observations.

\subsubsection{Beam smearing}

A natural question is whether the shallow slope of the curve of UGC 128 cannot be caused by beam-smearing effects, as the difference in physical resolution between the two curves is a factor of $\sim 15$. In other words, is UGC 128 a 
NGC 2403 in disguise? There are several reasons why it is very unlikely that this is the case.

We illustrate the effects of beam smearing by smoothing the rotation curve of NGC 2403 to the same physical resolution as that of UGC 128. This curve is shown as the light line in Fig. 1. It is obvious that even at this lower resolution the NGC 2403 curve is still different from the UGC 128 curve. The parameters of the maximum disk decomposition of this smoothed curve are also given in Table 1.

Note that this experiment is an extreme one: smoothing NGC 2403 to the UGC 128 physical resolution has a noticeable impact on $\Upsilon_{*}^{\max }$. Improving the resolution of UGC 128 to that of NGC 2403 probably will not have as big an effect: the domination of DM in LSB galaxies (see Sect. 3.3) produces smooth and featureless rotation curves that are relatively insensitive to beam smearing effects (de Blok \& McGaugh 1996). These curves do not show the characteristic tell-tale features of a dominant disk or bulge. This means that for halo-dominated galaxies the measured slope cannot be much different from the true slope.

Note that our exercise of smoothing the NGC 2403 curve to a lower resolution has only increased the discrepancy between the fits to the two curves as the difference in $\Upsilon_{*}^{\max }$ between the two galaxies has become larger.

\subsection{Masses of the components}

The values for the total masses of galaxies one derives from rotation curves are always lower limits. The flatness of the rotation curves shows that the cumulative mass keeps increasing with radius. When comparing masses of galaxies one thus has to measure the mass at a consistently defined radius to take this size-effect into account.

We derive the masses and mass-to-light ratios of both galaxies using three different definitions for this radius and show that all definitions result in UGC 128 being significantly more DM dominated than NGC 2403. These definitions are as follows: 1) The outermost measured radius $R_{\text {max }}$. This is the closest we can get to deriving the total mass, but as $R_{\max }$ is different for both galaxies, a significant size-effect is still present. Even at $R_{\max }$ one probably considerably underestimates the total mass. 2) A fixed number of scale lengths: in this case the masses within 6.2 scale lengths. This is the preferred way of measuring radii in a galaxy, as the scale length is intrinsic to a galaxy, and the only directly observable measure of the size. 3) A fixed number of kpc: in this case $20 \mathrm{kpc}$. This would be identical to definition 2 if all galaxies had identical surface brightnesses.

The radii were chosen to be as large as possible within the constraints of the observations and the respective definitions. Table 1 shows the values of the masses of the different components (gas, stars and DM) using the three different definitions.

The stellar masses have been computed by scaling the measured luminosities within the respective radii with $\Upsilon_{*}^{\max }$; the gas masses are also measured within the respective radii by scaling the relevant fraction of the HI mass with 1.4 to account for the helium. Finally, the dark masses have been computed using $M_{T}(R)=V(R)^{2} R / G$, where $R$ is the appropriate radius according to one of the definitions, and subtracting the visible mass.

In the cases of $R<R_{\max }$ and $R<6.2 h$ the dark component is much more dominant in the LSB galaxy (even under the assumption of maximum disk). The shapes and amplitudes of the rotation curves of other LSB galaxies (as presented in de Blok et al. 1996) strongly suggest that this is true for late-type LSB disk galaxies in general.

In the case of $R<20 \mathrm{kpc}$ the mass of the dark component in UGC 128 is here of the same order as that of the dark component in NGC 2403. It should be kept in mind, though, that $20 \mathrm{kpc}$ cor- 
responds to more than 9 scale lengths in NGC 2403 , that is, at $20 \mathrm{kpc}$ we are seeing the outermost observable parts of that galaxy. In UGC $12820 \mathrm{kpc}$ corresponds to only 2.9 scale lengths. This is well within the optical disk, where we are still sampling the rising part of the rotation curve. We are thus measuring two physically very different regions of the two galaxies. This result is therefore still consistent with the conclusion that DM is more dominant in LSB galaxies.

We want to stress that the maximum disk case is a worst case scenario. If, for example, $\Upsilon_{*}$ in UGC 128 is decreased to the value of $\Upsilon_{*}^{\max }$ in NGC 2403 (or a value appropriate for the stellar population), the contribution of the disk will naturally become less. The dark halo fit will have to adjust itself accordingly and put more DM into the inner disk. UGC 128 would thus become even more DM dominated.

\subsubsection{Mass-to-light ratios}

This brings us to the $M / L$ ratios: these are independent of any maximum disk assumptions, and are simply computed by dividing the mass $M_{T}(R)$ with the luminosity $L(R)$ (i.e. the mass and luminosity within radius $R$ ). As the luminosity of both galaxies is by choice equal, differences in the mass-to-light ratios are caused by differences in the total masses within each $R$. In all three cases UGC 128 has the highest value of $M / L$.

\subsubsection{Mass-surface densities}

This leaves the question of whether LSB galaxies have a lower baryonic mass surface density than HSB galaxies. That is, are LSB galaxies just normal surface density galaxies that happen to have an anomalously lower luminous surface density, or do they have intrinsically lower surface densities.

We illustrate this in Fig. 2. For both NGC 2403 and UGC 128 the radial surface density distributions of the gas and the stellar components are plotted, assuming the respective maximum disk $\Upsilon_{*}^{\max }$ values for the stellar disk. The surface densities shown are therefore the maximum values that can be accommodated within the constraints of the observed rotation curves. The total baryonic surface density in UGC 128 is still a factor of $\sim 5$ lower than that in NGC 2403, despite the maximum disk assumption, and despite the fact that $\Upsilon_{*}^{\max }$ is a factor of $\sim 2$ higher in the LSB galaxy.

Again, beam-smearing effects cannot cause these results. In Fig. 2 we also show the total baryonic surface density profile as derived from the maximum disk decomposition of the smoothed NGC 2403 curve. Even here, at identical physical resolutions, the baryonic surface density of UGC 128 is consistently a factor of 2 lower than in NGC 2403.

Fig. 2 illustrates an extreme case. If we relax the maximum disk assumption, then for all plausible assumptions UGC 128 will have the lowest baryonic mass surface density. One could of course assume maximum disk in UGC 128 and minimum disk in NGC 2403, but it should be obvious that this is unrealistic.

The rotation curve fitting procedure in most cases prefers a minimum disk solution for the LSB galaxies (de Blok \& McGaugh 1996), while the rotation curves of HSB galaxies are better fitted using a maximum disk solution. It is therefore unrealistic to lower the inferred baryonic surface densities of the HSB galaxy by lowering $\Upsilon_{*}^{\mathrm{HSB}}$, while at the same time keeping $\Upsilon_{*}^{\mathrm{LSB}}$ fixed at the maximum disk value. While maximum disk is thus a good working hypothesis for HSB galaxies, it is not in LSB galaxies.

We can thus conclude from Fig. 2 that both the gas surface density and the stellar disk mass surface density in UGC 128 are lower than those in NGC 2403. From the very similar shapes and amplitudes of the rotation curves and gas and surface brightness profiles (de Blok et al. 1996,1995; McGaugh \& Bothun 1994), we conclude that this is also true in general for LSB 
galaxies.

A detailed quantitative conclusion about the DM distribution would involve discussing the freedom for interplay between the various mass components during fitting. This is beyond the scope of this work. The halo parameters $\rho_{0}$ and $R_{C}$ in Table 1 are comparable, but are extremely sensitive to the assumed values of $\Upsilon_{*}^{\max }$. The different geometries of the disks and the fitted values of $\Upsilon_{*}$ tend to smooth out any intrinsic differences between the two halos by attributing them mostly to differences in $\Upsilon_{*}^{\max }$. However, a small difference still remains (Table 1), and becomes more pronounced at identical physical resolution. If the maximum disk assumption is relaxed, differences will become only more pronounced. For comparison we also show the halo parameters derived under the minimum disk assumption in Table 1.

A more robust statement is that the mean mass density enclosed by the two disks (at 6.2 scale lengths) differs by an order of magnitude: $\langle\rho\rangle=2.3 \times 10^{-3} M_{\odot} \mathrm{pc}^{-3}$ for UGC 128 and $\langle\rho\rangle=2.5 \times 10^{-2} M_{\odot} \mathrm{pc}^{-3}$ for NGC 2403 .

\section{Conclusions}

We have compared UGC 128 and NGC 2403, two galaxies at identical positions on the TF relation, but with a factor of 10 difference in surface brightness. Although the global properties $L$ and $V_{\max }$ are identical, the shape of the rotation curves differs with central surface brightness. The mass-to-light ratio of the LSB galaxy is always higher than that of the HSB galaxy at any given radius. This confirms the relation between surface brightness and mass-to-light ratio inferred from the TF relation (Zwaan et al. 1995). The surface densities of the gas and the stars in LSB galaxy UGC 128 are lower than in the HSB galaxy NGC 2403. The total mass density of UGC 128 is an order of magnitude lower than that of NGC 2403. If all of this applies to other LSB galaxies (as is strongly suggested by the shapes of their rotation curves [de Blok et al. 1996]), then LSB galaxies are true low density objects. 


\section{REFERENCES}

Athanassoula, E., Bosma, A., Papaioannou, S., 1987, A\&A, 179, 23

Begeman, K.G., 1987, PhD thesis, University of Groningen

Bottema, R., 1995, PhD thesis, University of Groningen

Broeils, A.H., 1992, PhD thesis, University of Groningen

de Blok W.J.G., McGaugh, S.S., 1996, in preparation

de Blok W.J.G., van der Hulst J.M., Bothun G.D., 1995, MNRAS, 274, 235

de Blok, W.J.G., McGaugh, S.S., van der Hulst, J.M., 1996, MNRAS, in press

Freeman K.C., 1970, ApJ, 160, 811

Kent S.M., 1987, AJ, 93, 816

Kormendy, J., 1990, in: "Evolution of the universe of galaxies; Proceedings of the Edwin Hubble Centennial Symposium", Astronomical Society of the Pacific, p. 33

Kuijken, K., Gilmore, G., MNRAS, 239, 571

McGaugh S.S., Bothun G.D., 1994, AJ, 107, 530

Roberts, M.S., Haynes, M.P., 1994, A\&AR, 32, 115

Tinsley, B.M., 1981, MNRAS, 194, 63

van Albada T.S, Sancisi R., 1986, Phil. Trans. R. Soc. Lond. A, 320, 447

van der Hulst J.M., Skillman E.D., Smith T.R., Bothun G.D., McGaugh S.S., de Blok W.J.G., 1993, AJ, 106, 548 (vdH93)

Zwaan M.A., van der Hulst J.M., de Blok W.J.G., McGaugh S.S., 1995, MNRAS, 273, L35
Fig. 1. - Comparison of the rotation curves of HSB galaxy NGC 2403 and LSB galaxy UGC 128. In the two panels the drawn lines are the observed rotation curves; the dotted lines represent the rotation curves of the gas components (observed Hi scaled by 1.4 to take He into account); the short dashed lines are the rotation curves of the disk, scaled to maximum disk; the long-dashed lines represent the rotation curves of the halos under the maximum disk assumption. The light dash-dotted lines in both panels show the rotation curve of NGC 2403 smoothed to the same physical resolution as the UGC 128 observations. Beam smearing cannot cause us to mistake one galaxy for the other.

Fig. 2.- Comparison of the surface densities of the baryonic matter in UGC 128 and NGC 2403. The stellar surface densities have been scaled by a factor $\Upsilon_{*}^{\max }$, as inferred from the maximum disk fits, and are therefore the maximum values both galaxies can accommodate within the constraints of their rotation curves. NGC 2403 is represented by the two full lines. The heavy top line shows the surface density inferred from the original rotation curve, the starred lower line that from the rotation curve smoothed to the physical resolution of that of UGC 128. The baryonic surface density in UGC 128 is significantly lower than that in NGC 2403.

This 2-column preprint was prepared with the AAS LATEX macros $\mathrm{v} 4.0$. 
TABLE 1

Comparison of UGC 128 and NGC 2403

\begin{tabular}{|c|c|c|}
\hline Name & UGC 128 & NGC 2403 \\
\hline$M_{B}(\mathrm{mag})$ & -18.9 & -19.2 \\
\hline$V_{\max }\left(\mathrm{km} \mathrm{s}^{-1}\right)$ & 131 & 136 \\
\hline$\mu_{0, B}\left(\operatorname{mag} \operatorname{arcsec}^{-2}\right)$ & 24.2 & 21.4 \\
\hline$h(\mathrm{kpc})$ & 6.8 & 2.1 \\
\hline$\Upsilon_{*, B}^{\max }$ & 3.0 & $1.8(0.8)$ \\
\hline$\rho_{0}^{\text {halo }}\left(\mathrm{M}_{\odot} \mathrm{pc}^{-3}\right)$ & $6.0 \times 10^{-3}$ & $10.2(19.9) \times 10^{-3}$ \\
\hline$R_{C}^{\text {halo }}(\mathrm{kpc})$ & 9.4 & $6.6(4.7)$ \\
\hline$\rho_{0}^{\text {halo,min disk }}\left(\mathrm{M}_{\odot} \mathrm{pc}^{-3}\right)$ & $21.7 \times 10^{-3}$ & $470 \times 10^{-3}$ \\
\hline$R_{C}^{\text {halo,min disk }}(\mathrm{kpc})$ & 4.0 & 0.8 \\
\hline$R_{\max }(\mathrm{kpc})$ & 42.3 & 19.5 \\
\hline$R_{\max } / h$ & 6.2 & 9.3 \\
\hline \multicolumn{3}{|c|}{$R<R_{\max }$} \\
\hline$M_{\text {gas }}\left(\times 10^{10} \mathrm{M}_{\odot}\right)$ & 1.05 & 0.43 \\
\hline$M_{\mathrm{star}}^{\max }\left(\times 10^{10} \mathrm{M}_{\odot}\right)$ & 1.68 & $1.39(0.61)$ \\
\hline$M_{\text {dark }}\left(\times 10^{10} \mathrm{M}_{\odot}\right)$ & 14.43 & $6.26(7.04)$ \\
\hline$\Upsilon_{B}^{\text {global }, R}$ & 30.6 & 10.5 \\
\hline \multicolumn{3}{|c|}{$R<6.2 h$} \\
\hline$M_{\text {gas }}\left(\times 10^{10} \mathrm{M}_{\odot}\right)$ & 1.05 & 0.34 \\
\hline$M_{\mathrm{star}}^{\max }\left(\times 10^{10} \mathrm{M}_{\odot}\right)$ & 1.68 & $1.36(0.60)$ \\
\hline$M_{\text {dark }}\left(\times 10^{10} \mathrm{M}_{\odot}\right)$ & 14.43 & $3.90(4.66)$ \\
\hline$\Upsilon_{B}^{\text {global }, R}$ & 30.6 & 7.4 \\
\hline \multicolumn{3}{|c|}{$R<20 \mathrm{kpc}$} \\
\hline$M_{\text {gas }}\left(\times 10^{10} \mathrm{M}_{\odot}\right)$ & 0.62 & 0.43 \\
\hline$M_{\mathrm{star}}^{\max }\left(\times 10^{10} \mathrm{M}_{\odot}\right)$ & 1.23 & $1.39(0.61)$ \\
\hline$M_{\text {dark }}\left(\times 10^{10} \mathrm{M}_{\odot}\right)$ & 5.41 & $6.26(7.04)$ \\
\hline$\Upsilon_{B}^{\text {global }, R}$ & 17.7 & 10.5 \\
\hline
\end{tabular}

Note.-Parenthesized values for NGC 2403 are results obtained from data smoothed to the physical resolution of UGC 128. 


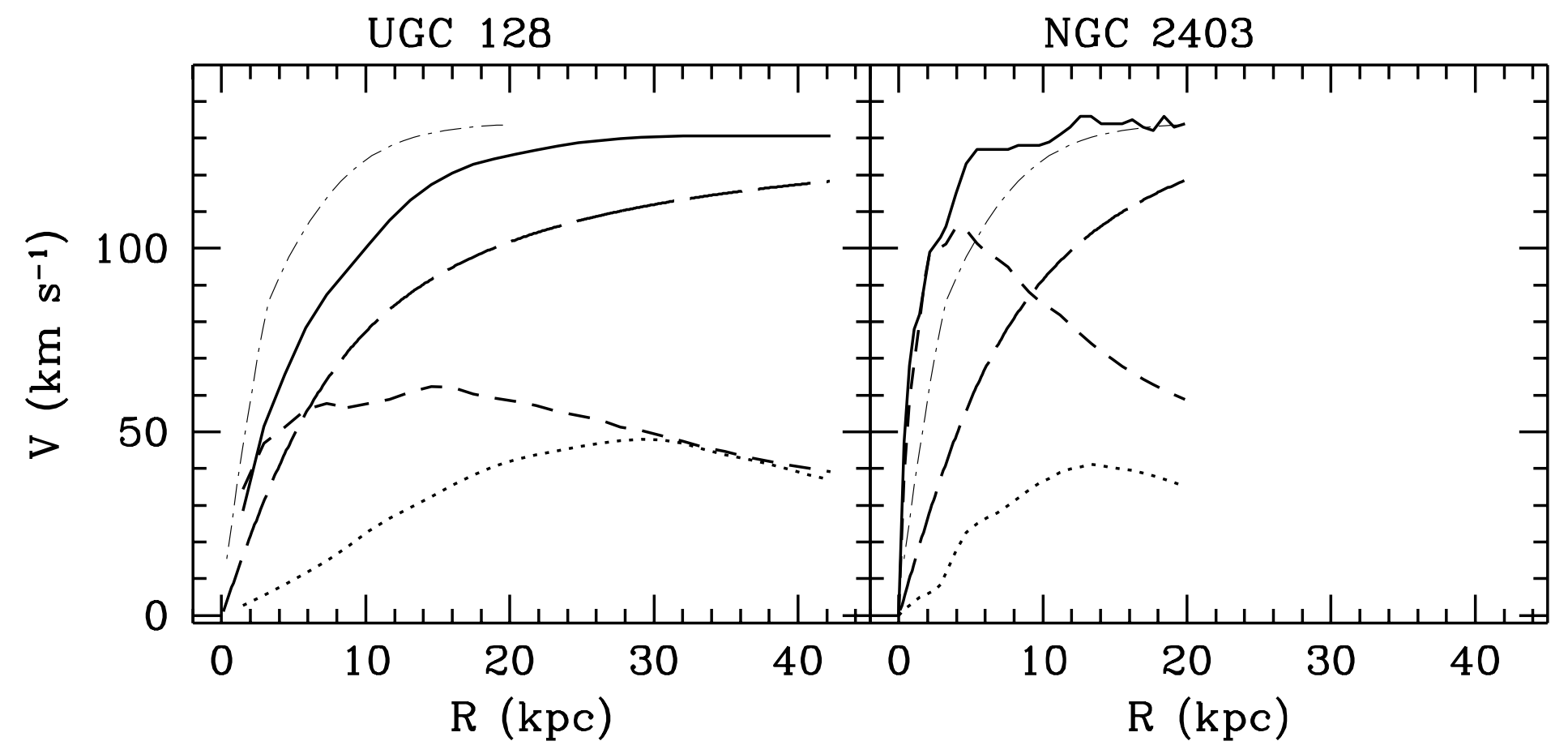




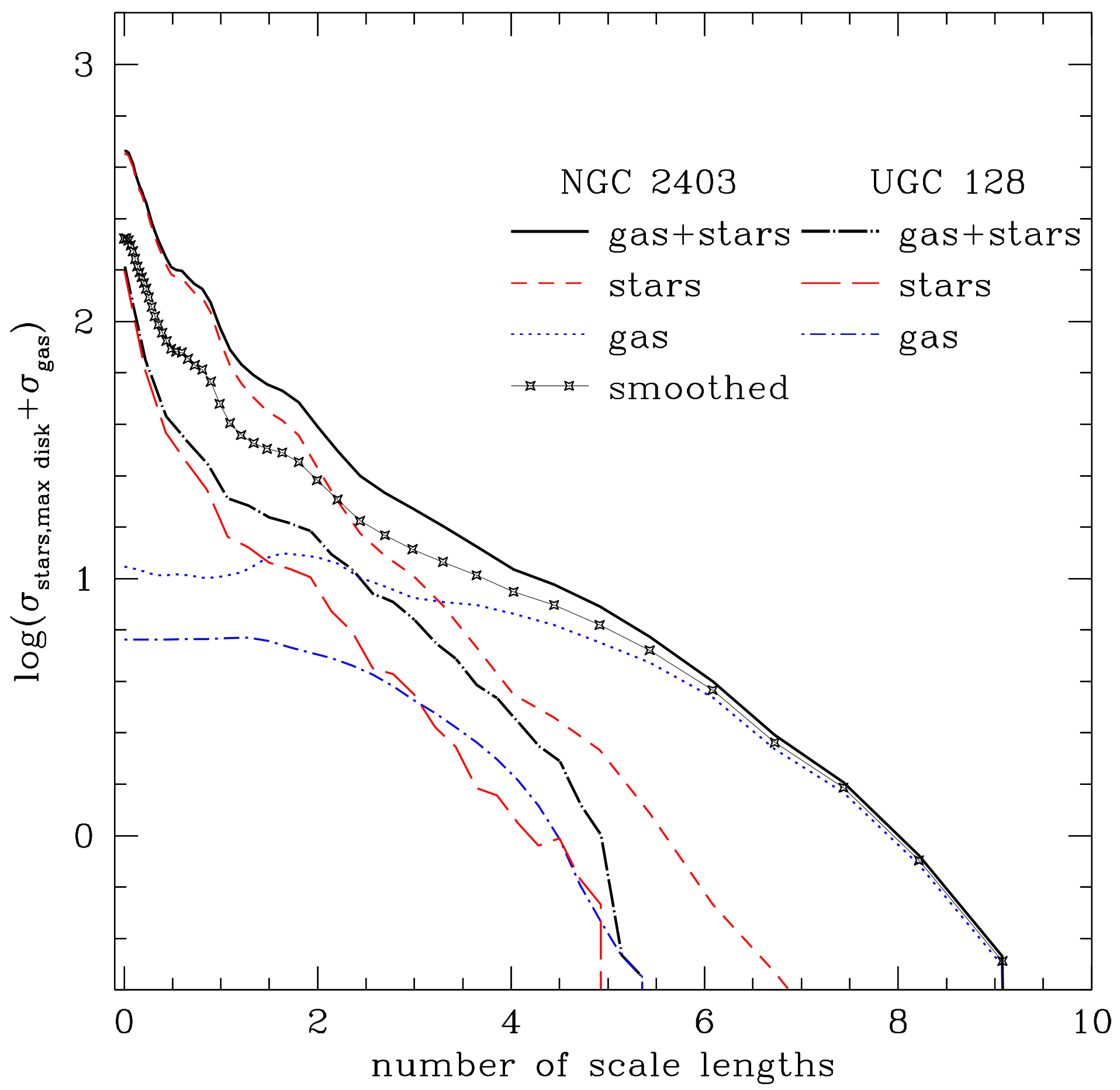

